\title{
Friuli-Venezia Giulia
}

\author{
Friuli-Venezia Giulia \\ Roberto Gasparini ${ }^{1}$, Donatella Panatto ${ }^{1}$, Bruna Dirodi ${ }^{2}$, Rosa Prato ${ }^{3}$, Gianni Amunni ${ }^{4}$, \\ Valter Turello ${ }^{5}$, Luigi Sudano ${ }^{6}$, Paolo Cristoforoni ${ }^{7}$, Sara Boccalini ${ }^{8}$, Paolo Bonanni ${ }^{8}$ \\ Dipartimento di Scienze della Salute, Università di Genova \\ 2 Direzione Access to Medicine, GlaxoSmithKline, Verona \\ 3 Dipartimento di Scienze Mediche e Chirurgiche, Università degli Studi di Foggia \\ 4 Dipartimento di Ginecologia, Perinatologia e Riproduzione Umana, Università di Firenze \\ 5 Dipartimento di Prevenzione U.O. Igiene e Sanità Pubblica, Azienda USL 3 Genova \\ 6 Assessorato Sanità, Salute e Politiche Sociali, Servizio Igiene, Sanità Pubblica, Veterinaria e degli Ambienti di lavoro \\ Dipartimento di Oncologia Ginecologica, Istituto Nazionale per la Ricerca sul Cancro, Genova \\ 8 Dipartimento di Sanità Pubblica, Università di Firenze
}

\begin{abstract}
In Friuli-Venezia Giulia region $86 \%$ of women (aged 24-64) are screened regularly, meaning every 3 years. The analysis on cross-protective activity exercised by bivalent and quadrivalent vaccines shows that the bivalent vaccine could prevent more pre-cancerous lesions and cases of cervicocarcinoma than quadrivalent, and that the latter could prevent genital warts that are not prevented by bivalent. The major number of cases avoided by the bivalent make it possible to fully offset the cost savings related to warts associated with the quadrivalent vaccine. Furthermore, a cost-effectiveness analysis shows that, considering regional tariffs, the multiple cohort (12-year-old +25 -year-old women) vaccination strategy with a $90 \%$ coverage could prevent 8 cases of cervicocarcinoma and 3 related deaths more than the vaccination of only 12-year-old girls, and thus proves to be cost-effective (11,960€/QALY).
\end{abstract}

\section{Keywords}

HPV; Vaccination strategy; Screening; Friuli-Venezia Giulia; Cost-effectiveness analysis

\section{IL CALENDARIO VACCINALE DELLA REGIONE FRIULI- VENEZIA GIULIA}

La regione Friuli-Venezia Giulia ha introdotto in passato nel suo calendario vaccinale anche la vaccinazione delle 15 enni come recupero delle coorti perse, che ha già portato a saldatura.

\section{RICORSO ALLO SCREENING}

Il dato di copertura di screening per la regione Friuli-Venezia Giulia è stato estrapolato dal report PASSI 2008 (Tabella I [1])

\section{ANALISI SULL'ATTIVITÀ DI CROSS-PROTEZIONE DEI VACCINI BIVALENTE E QUADRIVALENTE}

I risultati contenuti in Tabella II mostrano che entrambi i vaccini, bivalente e quadrivalente, apportano benefici clinici rispetto alla non vaccinazione. In particolare, si eviterebbero

\begin{tabular}{lc}
\hline \multicolumn{1}{c}{ Frequenza dello screening } & Tasso di copertura (\%) \\
\hline Regolare (ogni 3 anni) & 86 \\
Irregolare (> 3 anni) & 8 \\
Mai & 6 \\
\hline
\end{tabular}

Tabella I. Copertura di screening [1]

con il quadrivalente e il bivalente, rispettivamente da 87 a 114 casi di pap test anomali, da 576 a 592 lesioni CIN1, da 213 a 266 CIN2/3, da 46 a 52 casi di CCU (cancro della cervice uterina), e circa 530 casi di condilomi (questi ultimi dovuti esclusivamente all'azione del quadrivalente). I casi evitati in più dal bivalente di lesioni precancerose e CCU permetterebbero di compensare completamente i risparmi sui costi relativi ai condilomi associati al vaccino quadrivalente. Infatti i costi totali annui evitati sono di $€ 1.068 .230$ per il bivalente e $€ 1.014 .063$ per il quadrivalente.
Corresponding author Roberto Gasparini gasparini@unige.it

\section{Disclosure}

II presente supplemento è stato realizzato con

il finanziamento integrale di GlaxoSmithKline Spa 


\begin{tabular}{llccccc}
\hline & \multicolumn{1}{c}{$\begin{array}{c}\text { Tipo di } \\
\text { vaccino }\end{array}$} & $\begin{array}{c}\text { Pap test } \\
\text { anomali }\end{array}$ & CIN1 & Condilomi & CIN2/3 & Cancro \\
\hline Casi prevenibili (n/anno)* & & 312 & 1.186 & 624 & 346 & 60 \\
Costo unitario (€/caso) & Bivalente & 22,2 & 49,9 & 0,0 & 76,8 & 86,5 \\
Efficacia del vaccino (\%) & Quadrivalente & 17,0 & 48,5 & 84,3 & 61,5 & 76,5 \\
& Bivalente & 114 & 592 & 0 & 266 & 52 \\
Casi evitati (n) & Quadrivalente & 87 & 576 & 526 & 213 & 46 \\
& Bivalente & 9.208 & 181.070 & 0 & 263.418 & 614.535 \\
Costi evitati (€) & Quadrivalente & 7.051 & 176.172 & 76.681 & 211.092 & 543.068 \\
& Bivalente & $\mathbf{1 . 0 6 8 . 2 3 0}$ & & & & \\
\hline Totale costi evitati (€/anno) & Quadrivalente & $\mathbf{1 . 0 1 4 . 0 6 3}$ & & & & \\
& Differenza & $\mathbf{5 4 . 1 6 7}$ & & & & \\
\hline
\end{tabular}

Tabella II. Analisi dei costi dei vaccini bivalente e quadrivalente

* In base all'età di vaccinazione

Popolazione femminile 25-64 anni $=342.173$

\section{ANALISI SULL'INTRODUZIONE DI UNA SECONDA COORTE DI GIOVANI DONNE ADULTE}

\section{La campagna vaccinale}

La Tabella III confronta i dati per la vaccinazione di una coorte singola e di una coorte multipla.

\section{Costi}

I costi di screening e trattamento sono riportati nella Tabella IV.

In Tabella $\mathrm{V}$ sono riportati i benefici clinici e i risultati farmacoeconomici calcolati seguendo le singole coorti per tutta la vita.

Nelle prime due colonne sono indicati i casi che potrebbero essere evitati vaccinando solo le 12 enni e vaccinando le 12 enni +25 enni; l'ultima colonna rappresenta i benefici clinici evitati in più dalla vaccinazione delle due coorti, rispetto alla vaccinazione della singola coorte. Per la regione Friuli-Venezia Giulia si eviterebbero in più 82 lesioni CIN2/3, 8 casi di CCU e 3 morti da cervicocarcinoma, e se si

\begin{tabular}{lcc}
\hline Strategia vaccinale & Coorte singola & Coorte multipla \\
\hline Coorte da vaccinare & $12 \mathrm{enni}$ & 12enni $+25 \mathrm{enni}$ \\
Dimensione coorte $(n)^{*}$ & 5.065 & 10.091 \\
Copertura attesa $(\%)$ & 90 & 90 \\
\hline
\end{tabular}

Tabella III. Dati da considerare per l'ipotesi di introduzione di una seconda coorte di giovani donne adulte

* Dati Demo_ISTAT [2] per le ragazze al 12 e 25 anno di età, aggiornati al 1 gennaio 2011

\begin{tabular}{llc}
\hline \multicolumn{1}{c}{ Interventi } & \multicolumn{1}{c}{ Oggetto } & Costo $(\boldsymbol{\epsilon})$ \\
\hline $\begin{array}{l}\text { Screening e } \\
\text { trattamento CIN } \\
\text { (primo anno) }\end{array}$ & $\begin{array}{l}\text { Screening negativo } \\
\text { (pap test) }\end{array}$ & 17,00 \\
& $\begin{array}{l}\text { Trattamento CIN1 } \\
\text { Trattamento CIN2/3 }\end{array}$ & 272,60 \\
Follow up delle CIN & Follow up CIN1 & $1.053,75$ \\
(anni successivi) & Follow up CIN2/3 & 55,70 \\
Trattamento cancro & Cancro (media pesata & 111,40 \\
(complessivo) & dei vari stadi) & 14.004 \\
Vaccino & 1 ciclo completo di & $3 \times 50,00+7,25$ \\
& vaccinazione & (costo vaccino + costo \\
& somministrazione) \\
\hline
\end{tabular}

Tabella IV. Costi regionali dei trattamenti

\begin{tabular}{|c|c|c|c|c|}
\hline Benefici clinici & $\begin{array}{c}\text { Casi evitati } \\
\text { vaccinando le } \\
\text { 12enni } \\
\end{array}$ & $\begin{array}{c}\text { Casi evitati } \\
\text { vaccinando } \\
\text { 12enni + 25enni }\end{array}$ & $\begin{array}{c}\text { Benefici da } \\
\text { vaccinazione delle due } \\
\text { coorti (12enni + 25enni) }\end{array}$ & \\
\hline Casi di CCU (n) & 11 & 19 & 8 & \\
\hline Morti da CCU (n) & 5 & 8 & 3 & \\
\hline Lesioni CIN2/3 (n) & 152 & 234 & 82 & \\
\hline $\begin{array}{c}\text { Risultati } \\
\text { farmacoeconomici* }\end{array}$ & $\begin{array}{c}\text { Vaccinazione } \\
\text { 12enni }\end{array}$ & $\begin{array}{c}\text { Vaccinazione } \\
\text { 12enni + 25enni }\end{array}$ & Differenza & $\begin{array}{c}\text { Rapporto incrementale } \\
\text { (€/QALY) }\end{array}$ \\
\hline Costi totali $(€)$ & 1.371 .691 & 3.044 .855 & 1.673 .164 & \\
\hline Anni di vita guadagnati & 151,444 & 291,352 & 139,908 & 11.959 (ICER) \\
\hline QALY & 151,430 & 291,318 & 139,888 & 11.960 (ICUR) \\
\hline
\end{tabular}

Tabella V. Coorte singola: benefici clinici e analisi economica

* I risultati farmacoeconomici sono scontati annualmente del $3 \%$ 
considerano le modeste dimensioni delle coorti, questi numeri, seppur piccoli, assumono un significato importante.

In Tabella $\mathrm{V}$ è mostrato anche l'impatto finanziario della vaccinazione in termini di costi totali associati alla strategia screening + vaccinazione. Il maggior impegno di budget richiesto per la strategia multicoorte è da considerarsi sino a saldatura delle coorti, per poi continuare a vaccinare solo le 12enni. Anche prendendo in considerazione i valori di ICER (Incremental Cost-Effectiveness Ratio) e ICUR (Incremental Cost-Utility Ratio) si evidenzia come entrambi siano favorevoli all'aggiunta della seconda coorte.

\section{CONCLUSIONI}

Da entrambe le analisi effettuate si evince che la vaccinazione, sia essa indirizzata a una singola o multipla coorte, è costo-efficace e apporta numerosi benefici di salute in termini di riduzione delle lesioni precancerose e dei casi di CCU. La strategia vaccinale multicoorte implica un maggiore investimento economico da parte della regione Friuli-Venezia Giulia, ma le lesioni CIN2/3 e i casi di cervicocarcinoma evitati costituiscono, per il servizio sanitario regionale, un risparmio in termini di costi diretti associati alla patologia.

Infatti, come mostra la prima analisi, che fa riferimento a un periodo di un anno, allo steady state, cioè quando l'intera coorte sia stata vaccinata, le lesioni precancerose e i CCU evitati si traducono in termini economici in un risparmio importante di $€$ 1.068.230 dovuti al bivalente e $€ 1.014 .063$ dovuti al quadrivalente.

\section{BIBLIOGRAFIA}

1. Istituto Superiore di Sanità. Progressi delle Aziende Sanitarie per la Salute in Italia. Disponibile all'indirizzo: http://www.epicentro.iss.it/passi/sorvRisultatiRegionale.asp (ultimo accesso settembre 2012)

2. Istat. Demografia in cifre. Disponibile all'indirizzo: http://demo.istat.it/ (ultimo accesso settembre 2012) 